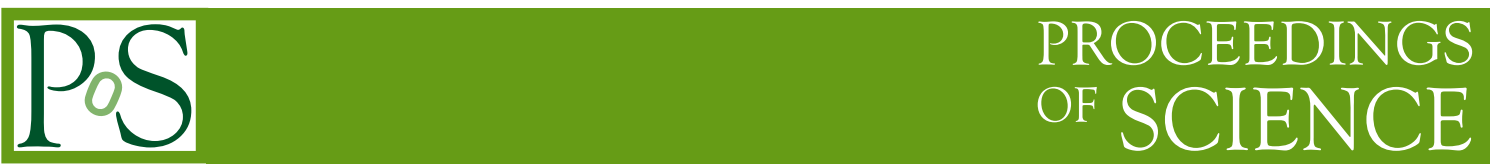

\title{
Euclidean 4D quantum gravity with a non-trivial measure term
}

\author{
Andrzej Görlich* \\ Niels Bohr Institute \\ E-mail: goerlichenbi.dk
}

Dynamical Triangulations provide us with a lattice regularization of four-dimensional Euclidean quantum gravity within the realm of ordinary quantum field theory. We add a local measure term, which can also serve as a generalized higher curvature term, and explore an extended coupling constant space. We determine the phase diagram of this model using non-degenerated triangulations. A first order phase transition line is observed, but no second order transition point is located. In consequence we cannot attribute any continuum physics interpretation to the so-called crinkled phase of $4 \mathrm{D}$ dynamical triangulations.

31st International Symposium on Lattice Field Theory LATTICE 2013

July 29 - August 3, 2013

Mainz, Germany

\footnotetext{
* Speaker.
} 


\section{Introduction}

The model of Dynamical Triangulations (DT) is based on the formalism of path integrals and lattice regularization [1, 2]. Its objective is to quantize a theory of gravitation. In the path integral formulation of quantum gravity, the role of a particle trajectory is played by the geometry of four-dimensional spacetime. Dynamical Triangulations define the way the path integral should be calculated and specifies the class of spacetime histories which should contribute to the path integral. The discretization appears only as a regularization intended to be removed in the continuum limit. No ad hoc discreetness of spacetime is assumed from the outset.

According to general theory of relativity, gravitation is encoded in spacetime geometry. The considered degree of freedom is the geometry associated with the metric field $g_{\mu v}(x)$. A nonzero curvature of the underlying spacetime geometry is interpreted as a gravitational field. The construction of the quantum theory starts from the gravitational path integral over geometries,

$$
Z=\int \mathrm{D}[g] e^{-S^{E H}[g]} .
$$

Dynamical Triangulations provide a lattice regularization of the formal functional integral and assumes that it can be represented via a sum over simplicial manifolds built of equilateral foursimplices,

$$
Z=\sum_{\mathscr{T}} e^{-S^{R}[\mathscr{T}]}
$$

A four-simplex is a generalization of a triangle to four dimensions, it is composed of five vertices connected to each other. The metric inside a simplex is flat. The four-simplices are glued pairwise along tetrahedral faces and the curvature is localized on triangles.

Because in DT all four-simplices are identical and equilateral, the classical Einstein-Hilbert action

$$
S^{E H}[g]=-\frac{1}{16 \pi G} \int \mathrm{d}^{4} x \sqrt{\operatorname{det} g}(R-2 \Lambda),
$$

has a very simple realization, the so-called Regge action,

$$
S^{R}[\mathscr{T}]=-\kappa_{2} N_{2}+\kappa_{4} N_{4},
$$

where $N_{2}$ is the number of triangles and $N_{4}$ number of four-simplices. Bare coupling constants $\kappa_{2}, \kappa_{4}$ are related to the bare Newton's constant $G$ and the bare cosmological constant $\Lambda$, respectively.

In the continuum limit, the bare cosmological constant has to be tuned to its critical value $\kappa_{4} \sim \kappa_{4}^{c}$. For $\kappa_{4}$ below the critical value the partition function is divergent. Effectively, such model has one coupling constants $\kappa_{2}$. Two non-physical phases were observed, namely the crumpled phase and branched polymers phase. The transition point turned out to be of first order [ []]. Usually, for critical systems on a lattice one can only associate continuum field theories to the fixed points if the transition is higher than first order.

\subsection{The measure term}

As DT is a lattice regularization of Euclidean geometries it is natural to consider enlarged coupling constant space involving higher curvature terms [凹]. Additional coupling constants may 
enrich the phase structure and lead to a continuum transition. It was shown that matter fields can prevent the collapse into branched polymers. For example, for more than 2 non-compact $U(1)$ gauge fields, the back-reaction of matter on geometry is strong enough to transform the branched polymer phase into smoother crinkled phase [6]. In the path integral (1.2) each triangulation carries the same weight. Even in the continuum it is not clear which measure $\mathrm{D}[g]$ should be chosen for the geometries. In the past, number of different choices of the measure for four-dimensional DT was proposed. A quasi-equivalence was shown in [6] between the model with gauge fields and with a modified measure,

$$
\sum_{\mathscr{T}} \rightarrow \sum_{\mathscr{T}} \prod_{t=1}^{N_{2}} o_{t}^{\beta}
$$

where $o_{t}$ is an order of triangle $t$. Such modified measure introduces additional parameter $\beta$. Recent work has renewed interest into probing the phase space of DT including such measure terms [7].

\subsection{The numerical setup}

Taking advantage of computer power we have today, we use Monte Carlo simulations to probe ensemble of combinatorial triangulations. Every four-simplex is uniquely defined by a set of 5 distinct vertices. and two adjacent four-simplices share exactly one face. The Monte Carlo algorithm uses a set of 5 ergodic local Pachner moves. We work in a pseudo-canonical ensemble of manifolds with topology $S^{4}$, and use the partition function

$$
Z\left(\kappa_{2}, \kappa_{4}, \beta\right)=\sum_{\mathscr{T}} \prod_{t=1}^{N_{2}} o_{t}^{\beta} \cdot e^{-\left[-\kappa_{2} N_{2}+\kappa_{4} N_{4}+\varepsilon\left(N_{4}-\bar{N}_{4}\right)^{2}\right]} .
$$

The quadratic term proportional to $\varepsilon$ fixes the total volume around some prescribed value $\bar{N}_{4}$. To achieve this the bare cosmological constant has to be tuned to its critical value $\kappa_{4} \approx \kappa_{4}^{c}$. To determine the phase diagram of this model we will be exploring the coupling constant space $\left(\kappa_{2}, \beta\right)$. For given values of the coupling constants, we will approximate the expectation values of observables $\mathscr{O}$ over Monte Carlo generated configurations:

$$
\langle\mathscr{O}\rangle_{\text {conf }}=\frac{1}{N_{\text {conf }}} \sum_{i=1}^{N_{\text {conf }}} \mathscr{O}_{i},
$$

where $N_{\text {conf }}$ is the number of independent configurations and $\mathscr{O}_{i}$ is the value of observable $\mathscr{O}$ calculated for the $i^{\text {th }}$ configuration.

\section{The phase diagram}

In order to determine the phase structure of the model we measured several observables such as the average number of triangles $\left\langle N_{2}\right\rangle$ and its susceptibility $\chi\left(N_{2}\right) \equiv\left(\left\langle N_{2}^{2}\right\rangle-\left\langle N_{2}\right\rangle^{2}\right) / N_{4}$. These observables have been used in the past to distinguish between the crumpled phase and the branched polymers phase. Another observable is the radius volume profile $V(r)$. We define a geodesic distance between two four-simplices as the length of the shortest path connecting these simplices. Each path consists of linear segments joining centers of neighboring simplices. Given a configuration $\mathscr{T}$ and a initial simplex $i_{0}, V\left(r, i_{0}, \mathscr{T}\right)$ denotes the number of simplices at geodesic distance 

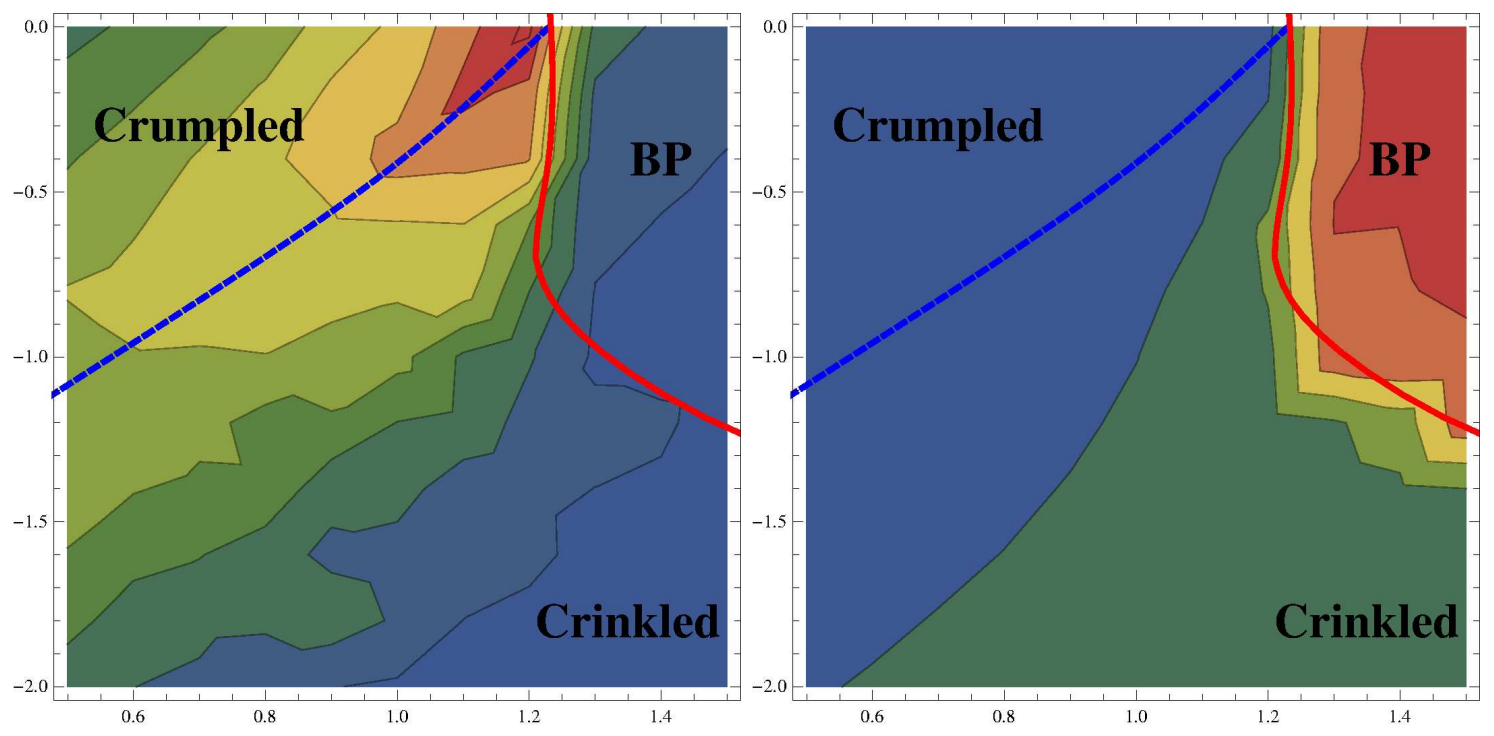

Figure 1: Density plots of the susceptibility $\chi\left(N_{2}\right)$ (left) and the average radius (right) in the $\kappa_{2}-\beta$ plane for $\left\langle N_{4}\right\rangle=160000$

$r$ from $i_{0}$. The average $V(r)$ over configurations and initial points, and the average radius $\langle r\rangle$ are then given by

$$
V(r) \equiv\left\langle\frac{1}{N_{4}} \sum_{i_{0}} V\left(r, i_{0}, \mathscr{T}\right)\right\rangle_{\mathscr{T}}, \quad\langle r\rangle \equiv \frac{1}{N_{4}} \sum_{r} r \cdot V(r) .
$$

We also look for the presence of so-called baby universes separated by minimal necks. A minimal neck correspond to the smallest nontrivial boundary of a four-dimensional simplicial manifold. It is a set of five tetrahedra, connected to each other, and forming a 4-simplex which is not present in the triangulation. Cutting the triangulation along a neck splits the triangulation into two separate parts. Because a minimal neck may contact other minimal necks, they equip triangulations with a graph structure.

\subsection{The grid}

The original approach of Euclidean Dynamical Triangulations corresponds to $\beta=0$. In this case there exist only crumpled phase and branched polymers phase, separated by a first order transition at $\kappa_{2} \approx 1.29$ [3]. At this point, we observe a peak of susceptibilities $\chi\left(N_{2}\right)$ and a jump in $\langle r\rangle$. There is also an abrupt change in baby universe structure, from collapsed graph (left of Fig. 2) to a fractal branched polymer structure (right of Fig. 2). The additional coupling constant $\beta$ may introduce new phases. We investigated a grid of points in the $\kappa_{2}-\beta$ plane with $\beta$ between 0 and -2 varied in steps of $\delta \beta=0.2$ and $\kappa_{2}$ between 0.5 and 1.5 varied in steps of $\delta \kappa_{2}=0.1$. This region embraces the transition point $\beta=0, \kappa_{2} \approx 1.29$. Plots of the susceptibility $\chi\left(N_{2}\right)$ (left) and the average radius (right) for the grid points ( $\kappa_{2}$ - horizontal axis, $\beta$ - vertical axis) are shown in Fig. 1. For negative $\beta$ the maximum of variance $\chi\left(N_{2}\right)$ (blue line) and a jump in $\langle r\rangle$ (red line) do not coincide any more. The branch polymer phase corresponds to large values of $\langle r\rangle$ and the peak of susceptibility is not a signal of phase transition. 


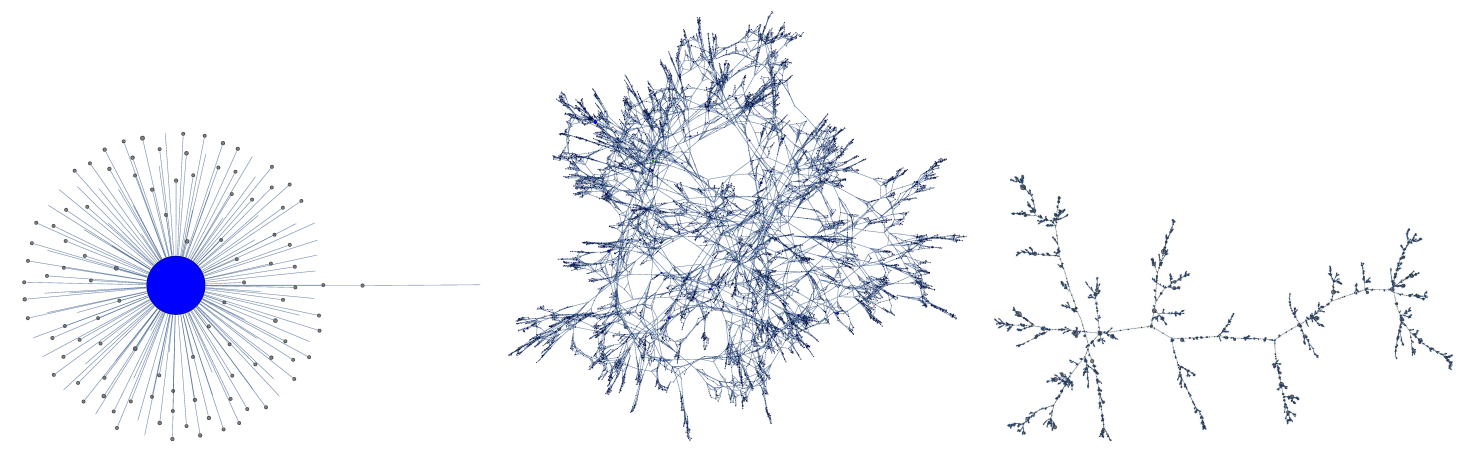

Figure 2: Minimal baby universe graph of a typical configuration in, respectively from left to right, crumpled phase, crinkled region and branched polymer phase.

We observe also a new hypothetical crinkled region where properties of typical configuration are between those in crumpled phase and branch polymer phase. It is located around point $\kappa_{2}=$ $2.0, \beta=-2.0$. The minimal baby universe structure in the crinkled region is very different from

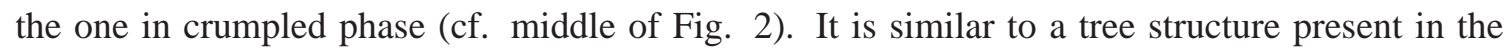
branched polymer phase, but contains loops which are identified with triangles of high order. Such triangles are not present in the two generic phases. The maximal order of triangles seem to behave like $\left\langle\operatorname{Max} o_{t}\right\rangle \propto N_{4}^{0.16}$. The transition from branched polymers to crinkled region is clear. The baby universe structure does not change abruptly, but there is a jump in $\langle r\rangle$ and a peak in $\chi\left(\log o_{t}\right)$ (cf. Fig. (4). Below, we outline properties of typical configurations from the branched polymers phase, the crumpled phase and the hypothetical crinkled region.

\begin{tabular}{|c|c|c|c|}
\hline & Branched polymers & Crumpled & Crinkled \\
\hline \hline Geometry & elongated & collapsed & between \\
\hline $\begin{array}{c}\text { Singular } \\
\text { sub-simplices }\end{array}$ & none & $\begin{array}{c}\text { two vertices, } o_{v} \propto N_{4} \\
\text { link of order } o_{l} \propto N_{4}^{2 / 3}\end{array}$ & $\begin{array}{c}\text { triangles of high order } \\
o_{t} \propto N_{4}^{0.16}\end{array}$ \\
\hline Baby universes & dominate & only small & many, but no large \\
\hline $\begin{array}{c}\text { Baby universe } \\
\text { structure }\end{array}$ & tree-like & collapsed & contains loops \\
\hline Hausdorff dimension & $d_{h}=2$ & $d_{h}=\infty$ & large or infinite \\
\hline Spectral dimension & $d_{s}=4 / 3$ & $d_{s}=\infty$ & large or infinite \\
\hline
\end{tabular}

\subsection{The path}

In order to verify the existence of the hypothetical crinkled phase mentioned before, we need to perform simulations for various total volumes and check scaling of observables. We follow a one-dimensional path shown in Fig. 3 (left). It starts at a point in crumpled phase and continuously leads through crinkled region to stop at branched polymers phase. If there is a phase transition between crumpled and crinkled phase, the path will have to cross it. As shown in Fig. 3, the path consists of three segments marked with different colors: a vertical segment I at $\kappa_{2}=0.5$, a horizontal segment II at $\beta=-2.0$, a vertical segment III at $\kappa_{2}=2.0$. The measurements were performed for three values of total volume $N_{4}=40 \mathrm{k}, 80 \mathrm{k}$ and $160 \mathrm{k}$. 

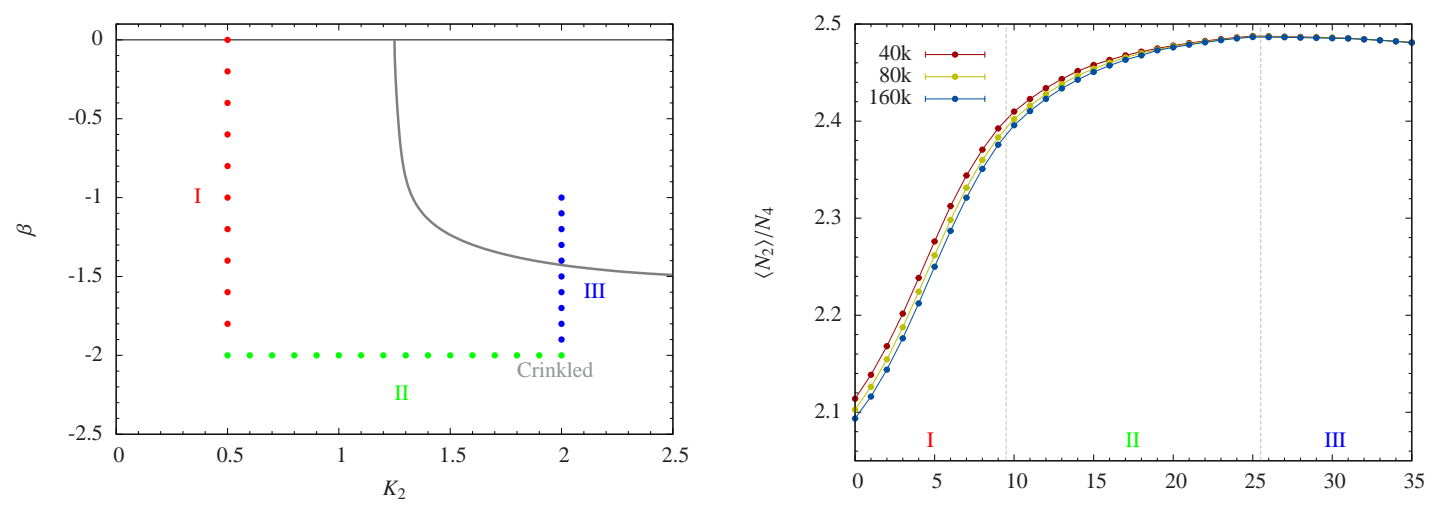

Figure 3: Left: A tentative phase diagram and a path (color points) from crumpled phase - through crinkled region - to branched polymer phase. The thick gray line denotes the phase transition between branched polymers and other phases, based on the grid measurements. Right: Plot of $\left\langle N_{2}\right\rangle / N_{4}$. Successive points of the path are on the $x$-axis.

When searching for a phase transition it is natural to look at parameters conjugate to coupling constants. The basic observable, scaled average number of triangles $\left\langle N_{2}\right\rangle / N_{4}$ is conjugate to $\kappa_{2}$ and is shown in Fig. 3 (right). The successive points on the path are presented on the $x$-axis and the vertical lines separate segments I, II and III. We do not observe any jump of $\left\langle N_{2}\right\rangle$ on the path between the crumpled phase and crinkled region. There is also no jump between the branched polymer phase and crinkled region, in contrast to $\beta=0$. However the scaling with $N_{4}$ changes exactly at the transition point (middle of segment III). Inside the branched polymer phase $\left\langle N_{2}\right\rangle \propto N_{4}$, while this scaling does not hold outside. Curves for different volumes diverge, as can be seen on the left side of Fig. B (right).

Another observable is an average logarithm of triangles' order, $\left\langle\log o_{t}\right\rangle$. Because $\left\langle\log o_{t}\right\rangle$ is conjugate to $\beta$, it increases when $\beta$ increases. The behavior of $\left\langle\log o_{t}\right\rangle$ is qualitatively similar to $\left\langle N_{2}\right\rangle$. The plot of the variance $\chi\left(\log o_{t}\right)=\left\langle\left(\log o_{t}\right)^{2}\right\rangle-\left\langle\log o_{t}\right\rangle^{2}$ is presented in Fig. 4 (left). There is a peak of the susceptibility $\chi\left(\log o_{t}\right)$ in segment $\mathrm{I}$, but it is decreasing with the total volume $N_{4}$ and can thus not be viewed as signaling a first or second order transition between the crumpled phase and hypothetical crinkled phase. While there are no other signals, it seems there is no phase transition associated with the maximum of susceptibility. There is also a small peak of the variance at the transition to branched polymer phase in segment III. It is not decreasing with the total volume and is a signal of the first order transition being a remnant of a peak at $\beta=0$. Behavior of $\chi\left(N_{2}\right)$ is similar to $\chi\left(\log o_{t}\right)$, but the latter peak is even less pronounced.

In branched polymer phase, the Hausdorff dimension $d_{h}=1 / 2$ and the average radius scales as $\langle r\rangle \propto N_{4}^{1 / 2}$. As shown in Fig. 1 and Fig. $\emptyset$ (right), in this phase $\langle r\rangle$ is relatively large. The jump of $\langle r\rangle$ at the boundary of branched polymer phase is a clear signal of a phase transition. Fig. $\theta$ shows that the jump of $\langle r\rangle$ becomes sharper as the total volume $N_{4}$ increases. There is no sign of any transition between crumpled and crinkled phase.

Following the path from crumpled phase to crinkled region, the baby universe graph dissolves gradually from one huge collapsed clump (left graph of Fig. 2) to a net structure (middle graph 

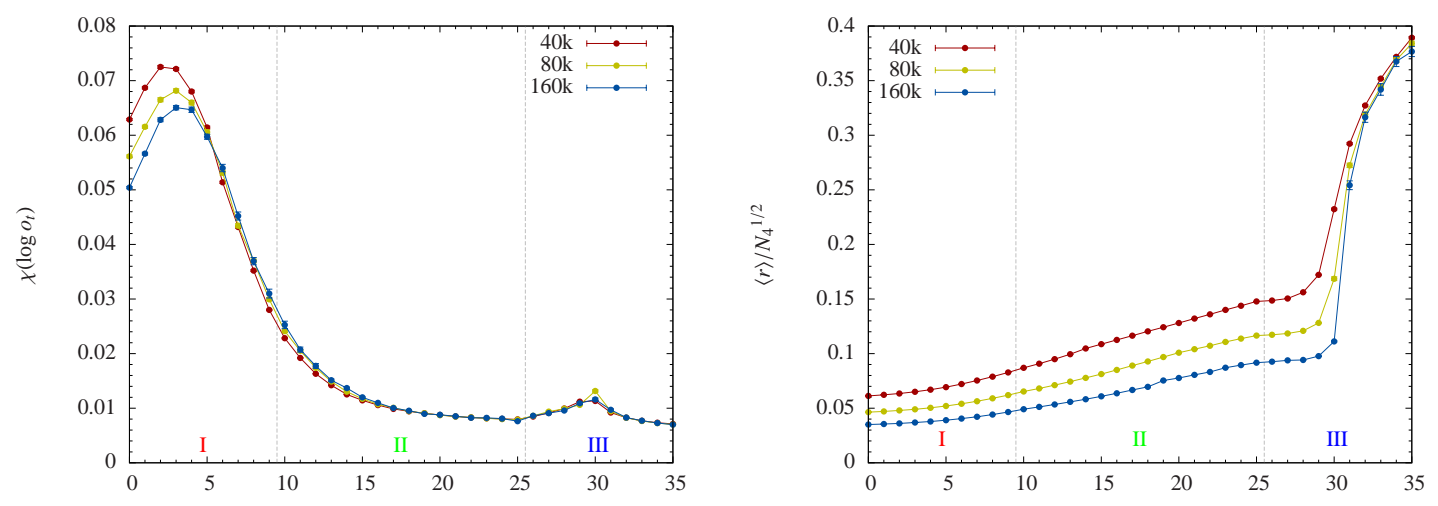

Figure 4: Left: Plot of $\chi\left(\log o_{t}\right)$ for points along the path. Right: Plot of $\langle r\rangle / N_{4}^{1 / 2}$ for points along the path.

of Fig. 2) without a distinct hub but with many loops, associated with triangles of high order. Although the baby universe structure is very different in the crumpled and crinkled region, we do not observe any abrupt change. When approaching the branched polymer phase, the loops - and high order triangles - disappear, and a tree-like fractal structure emerges (right graph of Fig. 2).

\subsection{Conclusions}

We were not able to observe a phase transition point where continuum physics is recovered. There is no signal, strengthening with the total volume, of a phase transition between crumpled phase and crinkled phase. Configurations in the crinkled region look more elongated $(V(r)$, minbu trees, spectral dimension), but the change is gradual when receding from the crumpled phase and it seems to be a finite size effect. We observed a first order phase transitions line at the boundary of the branched polymers phase. It is a continuation of the transition observed originally at $\beta=0$.

\section{References}

[1] J. Ambjorn, J. Jurkiewicz, Phys. Lett. B278 (1992) 42

[2] M. E. Agishtein and A. A. Migdal, Mod. Phys. Lett. A 7 (1992) 1039, Nucl. Phys. B 385 (1992) 395.

[3] P. Białas, Z. Burda, A. Krzywicki and B. Petersson, Nucl. Phys. B472 (1996) 293; B. de Bakker, Phys. Lett. B389 (1996) 238; S.M. Catterall, J.B. Kogut, R.L. Renken and G. Thorleifsson, Nucl. Phys. B53 (1997) 756

[4] J. Ambjorn, J. Jurkiewicz and C. F. Kristjansen, Nucl. Phys. B 393 (1993) 601, [hep-th/9208032].

[5] A. Görlich, Lect. Notes Phys. 863 (2013) 93; A. Görlich, Acta Phys. Pol. B 39, 3343 (2008)

[6] B. Brugmann, E. Marinari, Phys. Rev. Lett. 70 (1993) 1908; S. Bilke, Z. Burda, A. Krzywicki, B. Petersson, J. Tabaczek, G. Thorleifsson, Phys.Lett. B432 (1998) 279, Phys.Lett. B418 (1998) 266

[7] D. Coumbe, J. Laiho Phys.Rev.Lett. 107 (2011) 161301; 